J. Nonlinear Sci. Appl. 1 (2008), no. 1, 5-11

The Journal of $\mathbf{N}_{\text {onlinear }} \mathbf{S}_{\text {ciences and its }}$ A pplications

http://www.tjnsa.com

\title{
A NOT ON DOMINATING SET WITH MAPLE
}

\author{
M. MATINFAR ${ }^{1 *}$ AND S. MIRZAMANI $^{2}$
}

ABStRact. Let $\mathrm{G}$ be a $n$ - vertex graph. In 1996, Reed conjectured that $\gamma(G) \leq\lceil n / 3\rceil$ for every connected $3-\operatorname{regular} G$. In this paper, we introduce an algorithm in computer algebra system of MAPLE such that, by using any graph as input, we can calculate domination number $\gamma(G)$ and illustrated set of all dominating sets. It important that these sets choose among between $\left(\begin{array}{c}n \\ \gamma(G)\end{array}\right)$ sets.

\section{INTRODUCTION}

In a mathematician's terminology, a graph $G$ is a collection of points $V(G)$, and lines connecting some (possibly empty) subset of them, such that denoted by $E(G)$. The points of a graph are most commonly known as graph vertices. Similarly, the lines connecting the vertices of a graph are most commonly known as graph edges.

The most common type is graphs in which at most one edge (i.e., either one edge or no edges) may connect any two vertices. Such graphs are called simple graphs. If multiple edges are allowed between vertices, the graph is known as a multigraph. Vertices are usually not allowed to be self-connected, but this restriction is sometimes relaxed to allow such graph loops. Let $G$ be a loopless graph with vertex set $V(G)=\left\{v_{1}, \ldots, v_{n}\right\}$ and edge set $E(G)=\left\{e_{1}, \ldots, e_{m}\right\}$. The adjacency matrix of $G$ written $A(G)$, is the $n$-by- $n$ matrix in which entry $a_{i, j}$ is the number of edges in $G$ with endpoints $\left\{v_{i}, v_{j}\right\}$.

For a graph $G$ and a subset $S$ of the vertex set $V(G)$, denote by $N_{G}[S]$ the set of vertices in $G$ which are in $S$ or adjacent to a vertex in $S$. If $N_{G}[S]=V(G)$,

Date: Received: 2 March 2008.

* Corresponding author.

2000 Mathematics Subject Classification. 05C69; 05 C85.

Key words and phrases. Minimum dominating set. MDS. Maple. Adjacency matrix. 
then $S$ is said to be a dominating set (of vertices in $\mathrm{G}$ ). The domination number of a graph $G$, denoted $\gamma(G)$, is the minimum size of a dominating set of vertices in $G$.

Vizing conjectured that $\gamma(G) \gamma(H) \leqq \gamma(G \times H)$, where $G \times H$ is the graph product [?, ?]. While the full conjecture remains open, Clark and Suen [?] have proved the looser result $\gamma(G) \gamma(H) \leq 2 \gamma(G \times H)$. This is important, that we have procedure for calculate of $\gamma(G)$, moreover can see set of all dominating set of graph.

\section{MAPle Procedure}

In this section we describe Maple procedure for finding all elements of dominating set and $\gamma(G)$ with vertices and edges of a graph.

$>$ with(networks):

$>$ with(combinat, choose):

$>$ with(combinat, powerset):

$>$ with(linalg):

$>\mathrm{G}:=\operatorname{graph}(\{$ vertices $\},\{$ edges $\})$ :

For example (shown in Fig.1):Vertices $=\{1,2,3,4,5,6,7,8,9,10,11\}$ and edges $=\{\{1,2\}$, $\{2,3\},\{3,4\},\{4,1\},\{1,5\},\{3,8\},\{2,6\},\{2,7\},\{4,10\},\{4,9\},\{5,6\},\{5,10\},\{6,11\}$, $\{10,11\},\{7,8\},\{8,9\},\{7,11\},\{9,11\}\}$.

$>\mathrm{MM}:=\operatorname{adjacency}(\mathrm{G})$ :

$>\mathrm{V}:=\operatorname{vertices}(\mathrm{G})$ :

$>\mathrm{f}:=\operatorname{nops}(\mathrm{V})$ :

$>$ for $\mathrm{j}$ from 1 to $\mathrm{f}$ do

$>\mathrm{R}:=\operatorname{choose}(\mathrm{f}, \mathrm{j}): \mathrm{ff}:=\operatorname{nops}(\mathrm{R})$ :

$>$ for $t$ from 1 to ff do

$>$ flag: $=5$;

$>\mathrm{L}:=\mathrm{R}[\mathrm{t}]$

$>\mathrm{M}:=\mathrm{V}$ minus $\{\mathrm{L}[]\}$;

$>\mathrm{nn}:=\mathrm{f}-\mathrm{j}$

$>$ for $\mathrm{p}$ from 1 to nn do

$>\mathrm{S}:=0$

$>\mathrm{k}:=1$ :

$>$ while $k<=j$ do

$>\mathrm{S}:=\mathrm{S}+\mathrm{MM}[\mathrm{M}[\mathrm{p}], \mathrm{L}[\mathrm{k}]]$;

$>\mathrm{k}:=\mathrm{k}+1$

$>$ end do;

$>\mathrm{pp}:=\mathrm{p}$

$>$ if $\mathrm{S}=0$ then $\mathrm{p}:=\mathrm{nn}+1$; end if;

$>$ if $\mathrm{pp}=\mathrm{nn}$ and $S<>0$ then flag:=100; fflag:=flag; end if;

$>$ if flag=100 then $\operatorname{print}(\mathrm{L}, "$ is dominating $\operatorname{set}(\mathrm{MDS}) ")$; $\mathrm{jj}:=\mathrm{j}$; end if;

$>$ end do;

$>$ if fflag $=100$ and $t=f f$ then $\mathrm{j}:=\mathrm{f}+1$; end if;

$>$ end do; 
$>$ end do:

$>\operatorname{print}(" \gamma(G)=", \mathrm{jj})$;

\section{MAin RESUlts}

In this section with using of computer algebra system "MAPLE", we calculate $\gamma(G)$ and illustrated set of all dominating sets some famous graph.

Examples 1: A Hamiltonian circuit, is a graph cycle through a graph that visits each node exactly once. A graph possessing a Hamiltonian circuit is said to be a Hamiltonian graph and A graph that is not Hamiltonian is said to be nonhamiltonian. An $n$ - polyhedral graph is a 3-connected simple planar graph on nodes. The Herschel graph is the smallest nonhamiltonian polyhedral graph (shown in Fig. 1). It is the unique such graph on 11 nodes, and has 18 edges. In this graph, we can see

$$
\begin{gathered}
\gamma(G)=3 \text { and between }\left(\begin{array}{c}
11 \\
3
\end{array}\right)=165 \text { sets, set of all dominating sets is: } \\
S=\{[1,3,11],[1,8,11],[2,5,9],[2,8,10],[2,9,10],[3,5,11],[4,5,7],[4,6,7],[4,6,8]\} .
\end{gathered}
$$

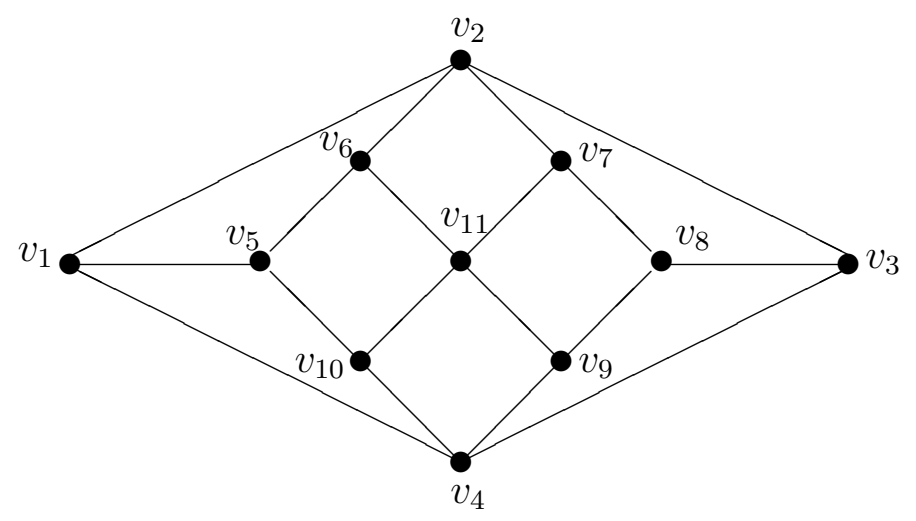

Fig. 1. The Hershel graph

Examples 2: The Petersen graph is the graph, (illustrated in Fig.2) in several

embeddings possessing ten nodes, all of whose nodes have degree three. In Petersen graph, we can see $\gamma(G)=3$ and and between $\left(\begin{array}{c}10 \\ 3\end{array}\right)=120$ sets, set of all dominating sets is:

$S=\{[1,3,8],[1,4,6],[1,9,10],[2,4,9],[2,5,7],[2,6,10],[3,5,10],[3,6,7],[4,7,8],[5,8,9]\}$. 


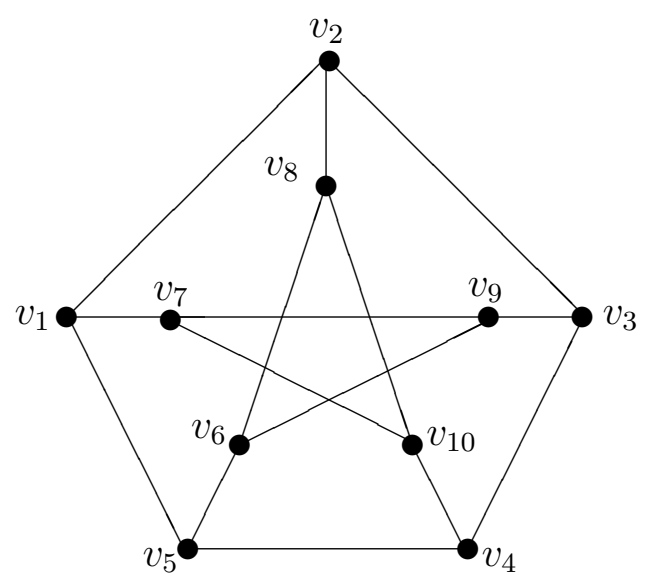

Fig. 2. The Petersen graph

Examples 3: A circulant graph is a graph of $n$ graph vertices in which the $i$ th graph vertex is adjacent to the $(i+j)$ th and $(i-j)$ th graph vertices for each $j$ in a list $l$. The circulant graph on $n$ vertices on a list of nodes $l$ is denoted by $C_{n}\langle l\rangle$. Thus, in Circulant graph $C_{8}\langle 1,4\rangle$, (shown in Fig. 3), we can see $\gamma\left(C_{8}\langle 1,4\rangle\right)=3$ and between $\left(\begin{array}{l}8 \\ 3\end{array}\right)=56$ sets, set of all dominating sets:

$$
\begin{aligned}
& S=\{[1,2,3],[1,2,8],[1,3,5],[1,3,6],[1,3,7],[1,4,6],[1,4,7],[1,5,7],[1,7,8],[2,3,4], \\
& {[2,4,6],[2,4,7],[2,4,8],[2,5,7],[2,5,8],[2,6,8],[3,4,5],[3,5,7],[3,5,8],[3,6,8], } \\
& {[4,5,6],[4,6,8],[5,6,7],[6,7,8]\} }
\end{aligned}
$$



Fig. 3. The Circulant graph

Examples 4: The degree of a graph vertex of a graph is the number of graph edges which touch the graph vertex, also called the local degree. A graph is said to be regular of degree $r$ if all local degrees are the same number $r$. In (Fig. 4), that is regular of degree 3 , we have $\gamma(H)=6$ and between $\left(\begin{array}{c}20 \\ 6\end{array}\right)=38760$ sets, set of all dominating sets is: $S=\{[1,3,7,9,15,20],[1,3,9,15,17,20],[1,3,10,15,17,20],[2,5,8,11,14,17]$, 
$[2,5,8,13,16,19],[2,5,9,11,14,17],[2,6,8,11,14,17],[2,6,9,11,14,16]$, $[2,6,9,11,14,17],[3,5,7,9,15,20],[3,5,8,11,14,17],[3,5,9,11,14,17]$, $[3,5,9,14,17,19],[3,5,9,14,17,20],[3,5,9,15,17,20],[3,6,8,11,13,17]$, $[3,6,8,11,14,17],[4,8,10,13,15,18],[5,7,10,12,15,19]\}$.

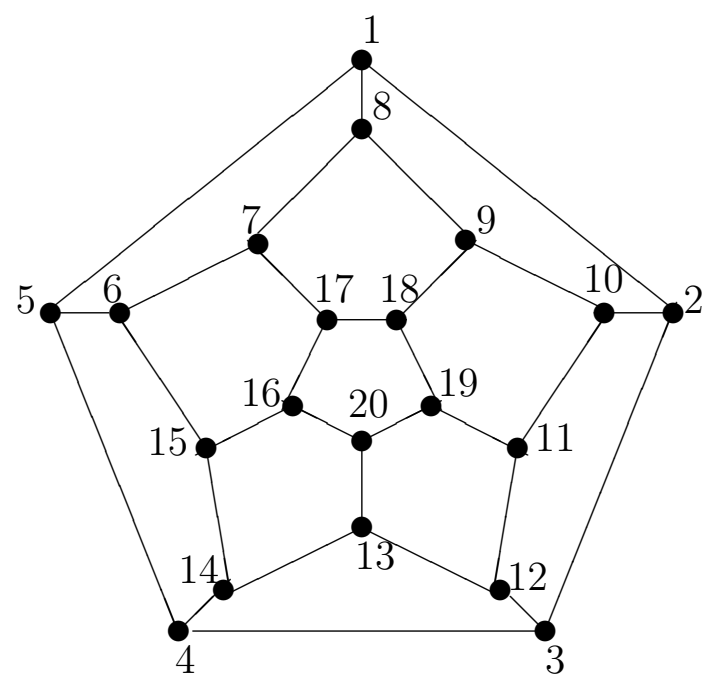

Fig. 4.

Examples 5: Harary graph $H_{k, n}$ is the smallest $k$-connected graph with $n$ graph vertices, having $\lceil k n / 2\rceil$ edges, where $\lceil x\rceil$ is the ceiling function. In $H_{3,18}$, (Fig.5) $\gamma\left(H_{3,18}\right)=5$ and between $\left(\begin{array}{c}18 \\ 5\end{array}\right)=9792$ sets, set of all dominating sets is:

$S=\{[1,2,6,8,13],[1,4,8,11,15],[1,4,8,12,15],[1,5,6,8,12],[1,5,8,12,14]$, $[1,5,8,12,15],[1,5,8,12,16],[1,6,8,12,13],[2,6,8,9,13],[2,6,9,13,16]$, $[2,6,9,13,17],[2,6,10,13,17],[3,6,10,13,17],[3,6,10,14,17],[3,7,10,14,17]$, $[4,7,11,15,18],[4,8,11,15,18]\}$,

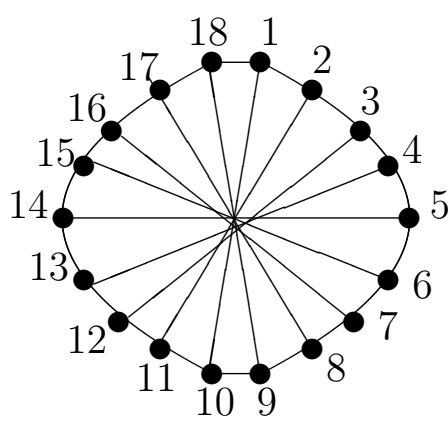

Fig. 5 . 
and in $H_{3,17}$ (following figure), $\gamma\left(H_{3,17}\right)=5$ and between $\left(\begin{array}{c}17 \\ 5\end{array}\right)=3315$ sets, set of all dominating sets is:

$$
\begin{aligned}
S=\{ & {[1,2,6,8,12],[1,2,6,12,15],[1,2,6,12,16],[1,4,8,10,14],[1,4,8,11,14], } \\
& {[1,5,6,8,11],[1,5,6,11,15],[1,5,6,11,16],[1,5,7,11,15],[1,5,8,11,13], } \\
& {[1,5,8,11,14],[1,5,8,11,15],[1,6,8,11,12],[1,6,11,12,15],[1,6,11,12,16], } \\
& {[2,6,8,9,12],[2,6,8,12,16],[2,6,8,12,17],[2,6,9,12,15],[2,6,9,12,16], } \\
& {[2,6,10,12,16],[2,6,12,15,17],[2,6,12,16,17],[3,4,8,9,14],[3,5,8,9,13], } \\
& {[3,6,8,9,12],[3,6,8,9,13],[3,6,9,12,15],[3,6,9,12,16],[3,6,9,13,15], } \\
& {[3,6,9,13,16],[3,7,8,9,13],[3,7,9,13,15],[3,7,9,13,16],[3,8,9,13,14], } \\
& {[4,7,10,14,17],[4,8,9,10,14],[4,8,10,14,17]\} . }
\end{aligned}
$$

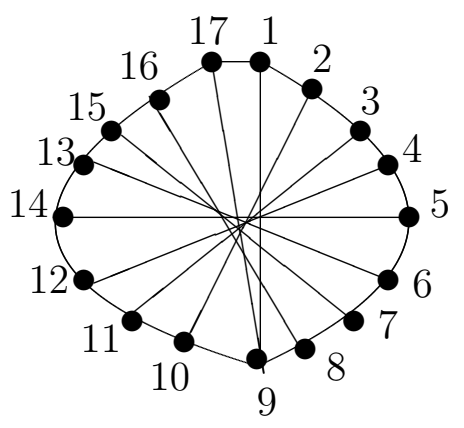

Fig. 6.

Examples 6: In 8-node tournament graph $C_{8}$, illustrated in (Fig. 6), we have $\gamma\left(C_{8}\right)=3$ and between $\left(\begin{array}{l}8 \\ 3\end{array}\right)=56$ sets, set of all dominating sets: $S=\{[1,3,6],[1,4,6],[1,4,7],[2,4,7],[2,5,7],[2,5,8],[3,5,8],[3,6,8]\}$.

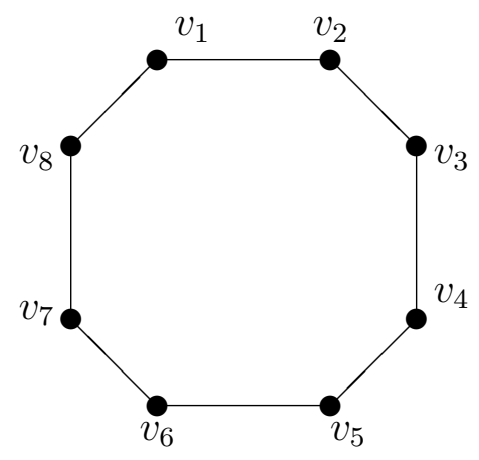

Fig. 6 
Examples 7: In final for particular graph $G$, that illustrated in (Fig. 7) we have $\gamma(G)=5$ and between $\left(\begin{array}{c}17 \\ 5\end{array}\right)=3315$ sets, set of all dominating sets is: $S=\{[1,2,5,7,10],[1,2,5,8,12],[1,2,5,10,13],[1,2,6,10,14],[1,2,7,10,15]$, $[1,2,8,12,15],[1,2,10,13,15],[1,3,5,7,11],[1,3,5,7,17],[1,3,5,11,13]$, $[1,3,5,13,17],[1,3,6,11,14],[1,3,6,12,16],[1,3,6,14,17],[1,3,7,11,15]$, $[1,3,7,15,17],[1,3,11,13,15],[1,3,13,15,17],[1,4,7,10,16],[1,4,8,11,14]$, $[1,4,8,12,16],[1,4,8,14,17],[1,4,10,13,16],[1,5,7,9,11],[1,5,7,9,17]$, $[1,5,9,11,13],[1,5,9,13,17],[1,6,9,11,14],[1,6,9,12,16],[1,6,9,14,17]$, $[1,7,9,11,15],[1,7,9,15,17],[1,9,11,13,15],[1,9,13,15,17],[2,3,7,11,15]$, $[2,5,9,13,17],[3,4,7,11,15],[3,6,7,11,15],[3,7,8,11,15],[3,7,10,11,15]$, $[3,7,11,12,15],[3,7,11,14,15],[3,7,11,15,16],[4,5,9,13,17],[5,6,9,13,17]$, $[5,8,9,13,17],[5,9,10,13,17],[5,9,12,13,17],[5,9,13,14,17],[5,9,13,16,17]\}$.

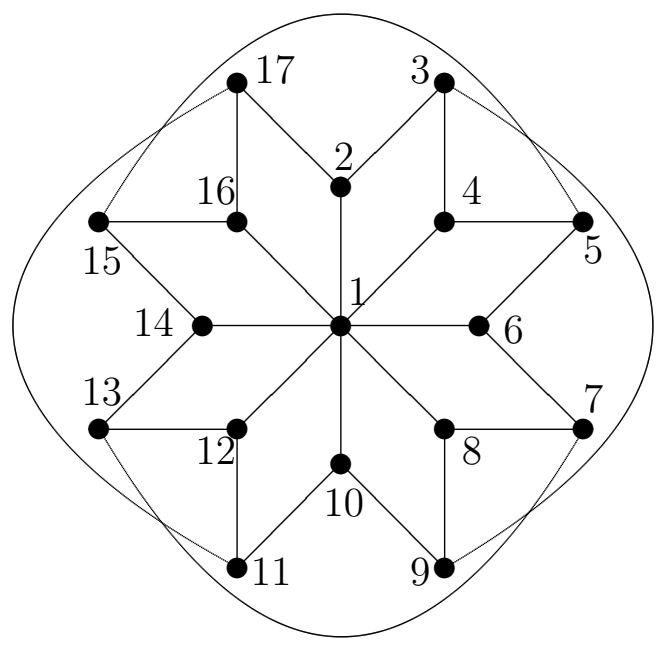

Fig. 7

REFERENCES

1. W. E. Clark, and S. Suen, An inequality related to Vizing's conjecture, Electron. J. Combin. 7 (2000), no. 1, Note 4, pp.3 (electronic).

2. B. Hartnell, and D. F. Rall, Domination in Cartesian Products: Vizing's Conjecture, Domination in Graphs-Advanced Topics, New York, Dekker,(1998), pp. 163-189.

3. T. W. Haynes, S. T. Hedetniemi, P. J. Slater, Domination in Graphs: Advanced Topics, Marcel Dekker, New York, Marcel Dekker, Inc. NewYork, (1998).

4. B. Read, Paths, stars, and the number three, combin. probab. comput.,5 (1996), 277-295.

1 Department of Mathematics, University of Mazandaran, P. O. Box 47416 1467, BABOLSAR, IRAN.

E-mail address: m.matinfar@umz.ac.ir

2 Department of Mathematics, University of Mazandaran, Babolsar, Iran. 